\title{
Determination of the Homotopy Type of a Morse-Smale Diffeomorphism on a 2-torus by Heteroclinic Intersection
}

\begin{abstract}
A. I. Morozov
According to the Nielsen - Thurston classification, the set of homotopy classes of orientationpreserving homeomorphisms of orientable surfaces is split into four disjoint subsets. Each subset consists of homotopy classes of homeomorphisms of one of the following types: $T_{1}$ ) periodic homeomorphism; $T_{2}$ ) reducible non-periodic homeomorphism of algebraically finite order; $T_{3}$ ) a reducible homeomorphism that is not a homeomorphism of algebraically finite order; $T_{4}$ ) pseudoAnosov homeomorphism. It is known that the homotopic types of homeomorphisms of torus are $T_{1}, T_{2}, T_{4}$ only. Moreover, all representatives of the class $T_{4}$ have chaotic dynamics, while in each homotopy class of types $T_{1}$ and $T_{2}$ there are regular diffeomorphisms, in particular, Morse-Smale diffeomorphisms with a finite number of heteroclinic orbits. The author has found a criterion that allows one to uniquely determine the homotopy type of a Morse-Smale diffeomorphism with a finite number of heteroclinic orbits on a two-dimensional torus. For this, all heteroclinic domains of such a diffeomorphism are divided into trivial (contained in the disk) and non-trivial. It is proved that if the heteroclinic points of a Morse-Smale diffeomorphism are contained only in the trivial domains then such diffeomorphism has the homotopic type $T_{1}$. The orbit space of non-trivial heteroclinic domains consists of a finite number of two-dimensional tori, where the saddle separatrices participating in heteroclinic intersections are projected as transversally intersecting knots. That whether the Morse-Smale diffeomorphisms belong to types $T_{1}$ or $T_{2}$ is uniquely determined by the total intersection index of such knots.
\end{abstract}

Keywords: Morse-Smale diffeomorphisms, Nielsen-Thurston theory, heteroclinic intersections, homotopy class of a map

\section{Introduction and formulation of the results}

Let $X$ be a topological space. Two continuous maps $f_{0}, f_{1}: X \rightarrow X$ are called homotopic (denoted by $f_{0} \simeq f_{1}$ ), if there is a continuous map $H: X \times[0,1] \rightarrow X$ such that $H(x, 0)=$

Received December 04, 2021

Accepted December 13, 2021

This work was supported by a grant from the Russian Science Foundation, contract 21-11-00010.

Andrei I. Morozov

aimorozov@hse.ru

National Research University Higher School of Economics

ul. B. Pecherskaya 25/12, Nizhny Novgorod, 603150 Russia

RUSSIAN JOURNAL OF NONLINEAR DYNAMICS, 2021, 17(4), 465-473 
$=f_{0}(x)$ and $H(x, 1)=f_{1}(x)$. A map $H$ is called a homotopy between $f_{0}$ and $f_{1}$. For every fixed $t \in[0,1]$ we set $H(x, t)=H_{t}(x)$. Denote by $[f]$ the homotopy class of the map $f$, that is $f^{\prime} \in[f] \Longleftrightarrow f^{\prime} \simeq f$.

The set of homotopy classes of homeomorphisms of the space $X$ forms the group $\Gamma(X)$ with the group operation $\left[f_{1}\right] \circ\left[f_{2}\right]=\left[f_{1} \circ f_{2}\right]$, which is called mapping class group. Thus,

$$
\Gamma(X): \stackrel{\text { def }}{=} \operatorname{Homeo}(X) / \simeq \text {, }
$$

where $\operatorname{Homeo}(X)$ is the set of homeomorphisms of the space $X$ onto itself.

A homotopy $H_{t}: X \rightarrow X, t \in[0,1]$ is called an isotopy if, for any $t$ the map $H_{t}$ is a homeomorphism of the space $X$.

According to the Nielsen - Thurston classification [1] there are four types of homotopy classes of surface homeomorphisms. Each subset contains only homotopy classes of homeomorphisms of one of the following types:

$T_{1}$ ) periodic homeomorphism;

$T_{2}$ ) reducible non-periodic homeomorphism of algebraically finite order;

$T_{3}$ ) reducible homeomorphism that is not a homeomorphism of algebraically finite order;

$T_{4}$ ) pseudo-Anosov homeomorphism.

Let $M^{2}$ be a connected compact (possibly with boundary) orientable surface of genus $g$. Recall that a homeomorphism $h: M^{2} \rightarrow M^{2}$ is called a periodic homeomorphism, if there exists $m \in \mathbb{N}$, such that $h^{m}=i d$, where $i d$ is the identity transformation. The smallest such number $m$ is called the period of the periodic homeomorphism.

A homeomorphism $h: M_{2} \rightarrow M_{2}$ is called a reducible by a system $C$ of disjoint simple closed curves $C_{i}, i=1, \ldots, l$, non-homotopic to zero and pairwise non-homotopic to each other if the system of curves $C$ is invariant under $h$.

A reducible non-periodic homeomorphism $h: M^{2} \rightarrow M^{2}$ is called a homeomorphism of algebraically finite order, if there exists an $h$-invariant neighbourhood $\mathbb{C}$ of curves of the set $C$, consisting of the union two-dimensional annuli and such that for each connected component $M_{j}^{2}$, $j=1, \ldots, n$ of the set $M^{2} \backslash$ int $\mathbb{C}$ there is a number $m_{j} \in \mathbb{N}$ such that $\left.h^{m_{j}}\right|_{M_{j}^{2}}: M_{j}^{2} \rightarrow M_{j}^{2}$ is a periodic homeomorphism.

Since the Nielsen-Thurston theory was originally described for surfaces of genus $g \geqslant 2$, it is worth mentioning the book [2], in which this theory is also generalized to a torus and a fact is presented that the homotopic types of homeomorphisms of torus are $T_{1}, T_{2}, T_{4}$ only (Theorem 13.1, p. 369).

Recall that the diffeomorphism $f: M^{n} \rightarrow M^{n}$, given on a smooth, closed, connected $n$-manifold $(n \geqslant 1) M^{n}$ is called Morse-Smale diffeomorphism, if

1) the non-wandering set $\Omega_{f}$ consists of a finite number of hyperbolic orbits;

2) the manifolds $W_{p}^{s}, W_{q}^{u}$ intersect transversally for any non-wandering points $p, q$.

In the set of periodic orbits of such a diffeomorphism, one can introduce a complete order relation, which is an extension of S. Smale's partial order [3]. Namely, let $\mathcal{O}_{i}, \mathcal{O}_{j}$ be the periodic orbits of the Morse-Smale diffeomorphism $f$. One can say that the orbits $\mathcal{O}_{i}, \mathcal{O}_{j}$ are in the relation $\prec\left(\mathcal{O}_{i} \prec \mathcal{O}_{j}\right)$, if $W_{\mathcal{O}_{i}}^{s} \cap W_{\mathcal{O}_{j}}^{u} \neq \varnothing$. A sequence of different periodic orbits $\mathcal{O}_{i}=\mathcal{O}_{i_{0}}$, $\mathcal{O}_{i_{1}}, \ldots, \mathcal{O}_{i_{k}}=\mathcal{O}_{j}(k \geqslant 1)$, such that $\mathcal{O}_{i_{0}} \prec \mathcal{O}_{i_{1}} \prec \ldots \prec \mathcal{O}_{i_{k}}$ is called a chain of the length $k$, connecting periodic orbits $\mathcal{O}_{i}$ and $\mathcal{O}_{j}$. The chain connecting the periodic orbits of saddle points will be called saddle chain. Due to the finiteness of the non-wandering set, for any diffeomorphism $f \in M S\left(M^{n}\right)$ there is a well-defined number equal to the length of the maximal saddle chain, which is denoted by beh(f). 
Denote by $M S_{1}\left(\mathbb{T}^{2}\right)$ the set of orientation-preserving Morse-Smale diffeomorphisms $f$ defined on the two-dimensional torus $\mathbb{T}^{2}$ and having $b e h(f) \leqslant 1$. According to $[4,5]$ the MorseSmale diffeomorphism with beh $(f) \leqslant 1$ exists in both the first $T_{1}$ and the second $T_{2}$ NielsenThurston type. We state $F=f^{d}: \mathbb{T}^{2} \rightarrow \mathbb{T}^{2}$, where $d \in \mathbb{N}$ is the smallest number such that $\forall x \in \Omega_{f}: f^{d}(x)=x$ and the map $\left.f\right|_{W_{x}^{u}}$ preserves orientation. The dynamics of any diffeomorphism $F \in M S_{1}\left(\mathbb{T}^{2}\right)$ can be represented as follows. The set $\Omega_{F}$ of periodic orbits of the map $F$ can be splatted into subsets $\Omega_{F}^{i}, i \in\{\omega, s, u, \alpha\}$ :

- $\Omega_{F}^{\omega}$ - the set of all sink orbits;

- $\Omega_{F}^{s}$ - the set of saddle orbits, whose unstable sets do not contain heteroclinic points;

- $\Omega_{F}^{u}$ - the set of the remaining saddle orbits of the system;

- $\Omega_{F}^{\alpha}$ - the set of source orbits.

Let

$$
\mathcal{A}_{F}=\Omega_{F}^{\omega} \cup W_{\Omega_{F}^{s}}^{u}, \quad \mathcal{R}_{F}=\Omega_{F}^{\alpha} \cup W_{\Omega_{F}^{u}}^{s}, \quad V_{F}=\mathbb{T}^{2} \backslash\left(\mathcal{A}_{F} \cup \mathcal{R}_{F}\right) .
$$

By virtue of the paper [6], the sets $\mathcal{A}_{F}$ and $\mathcal{R}_{F}$ are an attractor and a repeller of the diffeomorphism $F$, respectively. All heteroclinic points of the diffeomorphism $F$ belong to the set $V_{F}$, which consists of a finite number of connected components $V_{i}, i=1, \ldots, m$. Each component $V_{i}$ is homeomorphic to an open two-dimensional annuli (see, for example [7]) and has a fundamental domain $K_{i} \cong[0,1] \times \mathbb{S}^{1}$, bounded by the circles $b_{i} \cong\{0\} \times \mathbb{S}^{1}, F\left(b_{i}\right) \cong\{1\} \times \mathbb{S}^{1}$, so that

$$
V_{i}=\bigcup_{n \in \mathbb{Z}} F^{n}\left(K_{i}\right)
$$

The component $V_{i}$ of the set $V_{F}$ will be called trivial, if the curve $b_{i}$ is homotopic to zero on the torus, and non-trivial otherwise. The component $V_{i}$ of the set $V_{F}$ will be called a heteroclinic domain, if the component $V_{i}$ contains at least one heteroclinic orbit.

The following lemma is proved in section 2 .

Lemma 1. If all heteroclinic domains of a diffeomorphism $F$ are trivial, then the diffeomorphism $F$ is isotopic to the identity map and, therefore, the homotopy class $[f]$ is of the type $T_{1}$.

In what follows, assume that the diffeomorphism $F$ has non-trivial heteroclinic domains. Let us renumber the connected components of the set $V_{F}: V_{i}, i=1, \ldots, m$ in such a way that the first $m_{0}$ components are non-trivial heteroclinic domains, and the rest are trivial.

Consider the covering of the torus $q: \mathbb{R}^{2} \rightarrow \mathbb{T}^{2}$, representing the torus as a quotient group of the plane $\mathbb{R}^{2}$ by the group of integer shifts. Let

$$
a=q(O x), \quad b=q(O y) .
$$

Then the curves $a, b$ are generators on the torus with a positive traversal direction inherited from the coordinate axes, and their homotopy classes are $[a]=\langle 1,0\rangle,[b]=\langle 0,1\rangle$. Then the homotopy class $[c]$ of any simple closed curve $c \in \mathbb{T}^{2}$ is determined by two numbers $[c]=\langle\mu, \nu\rangle$, $\mu \in \mathbb{Z}, \nu \in \mathbb{N}$, where $\mu, \nu$ denote the number of revolutions around the generators $a, b$. Moreover, the numbers $\mu, \nu$ are coprime, that is, $(\mu, \nu)=1$.

Without loss of generality (see, for example, [8]), we assume that all curves $b_{i}, i \in\left\{1, \ldots, m_{0}\right\}$ are of the form $\left\{y_{i}\right\} \times b$, and the generator $a$ intersects the annuli $K_{i}$ along the segment $a_{i}$, connecting the point $Y_{i}=y_{i} \times\{0\}$ with the point $F\left(Y_{i}\right)$. We state $\widehat{V}_{i}=V_{i} / F=K_{i} / F$ is the quotient 
space by the action of the diffeomorphism $F$. Denote by $p_{i}: V_{i} \rightarrow \widehat{V}_{i}$ the natural projection, which is also a covering map for the space $\widehat{V}_{i}$. Then (see, for example, [7]) $\widehat{V}_{i}$ is homeomorphic to the two-dimensional torus and the curves $\widehat{a}_{i}=p_{i}\left(a_{i}\right), \widehat{b}_{i}=p_{i}\left(b_{i}\right)$ are generators on $\widehat{V}_{i}$ such that $\left[\widehat{a}_{i}\right]=$ $=\langle 1,0\rangle$ and $\left[\widehat{b}_{i}\right]=\langle 0,1\rangle$ (see, Fig. 1 ), while the orientations on $a_{i}$ and $b_{i}$ are induced by the orientations on $a$ and $b$.

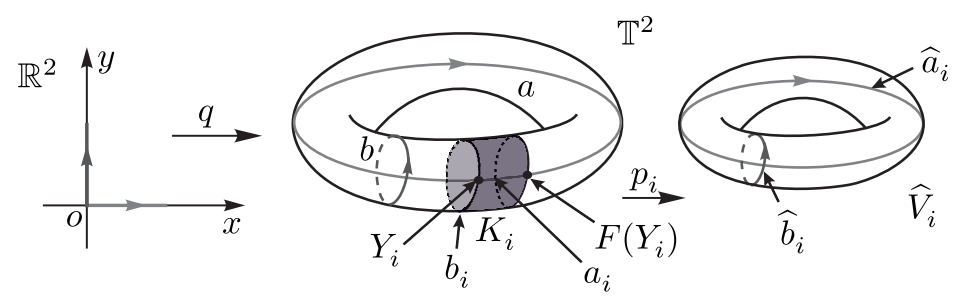

Fig. 1. The projection $q$ defines generators $a, b$ on the torus $\mathbb{T}^{2}$ as images of the axes $O x$ and $O y$. The projection $p_{i}$ defines the generators $\widehat{a}_{i}, \widehat{b}_{i}$ on the torus $\widehat{V}_{i}$ as the images of the segments $a_{i}, b_{i}$. The connection between the basis of the torus $\mathbb{T}^{2}$ and of the torus $\widehat{V}_{i}$ is established in a natural way using the map $p_{i}$ for all $i \in 1, \ldots, m_{0}$

Denote by $L_{i}^{s}\left(L_{i}^{u}\right)$ the union of the stable (the unstable) separatrices belonging to the domain $V_{i}$. Let $\widehat{L}_{i}^{s}=p_{i}\left(L_{i}^{s}\right)\left(\widehat{L}_{i}^{u}=p_{i}\left(L_{i}^{u}\right)\right)$. Denote by $r_{i}^{s}$ and $r_{i}^{u}$ the number of curves in the sets $\widehat{L}_{i}^{s}$ and $\widehat{L}_{i}^{u}$ respectively. Since the curves of the set $\widehat{L}_{i}^{s}\left(\widehat{L}_{i}^{u}\right)$ are pairwise disjoint and $F=f^{d}$, then any curve $\widehat{l}_{i}^{s} \subset \widehat{L}_{i}^{s}\left(\widehat{l}_{i}^{u} \subset \widehat{L}_{i}^{u}\right)$ is of homotopy type $\left\langle\delta_{i}, d_{i}^{s}\right\rangle\left(\left\langle\delta_{i}, d_{i}^{u}\right\rangle\right)$, where $\delta_{i}= \pm 1$ and $d$ is a multiple of $d_{i}^{s}\left(d_{i}^{u}\right)$. Since the separatrices are oriented in a natural way from the repeller to the attractor, the number $\delta_{i}$ always matches for $\widehat{l}_{i}^{s}$ and $\widehat{l}_{i}^{u}$. For any two curves $\widehat{l}_{i}^{s} \subset \widehat{L}_{i}^{s}, \widehat{l}_{i}^{u} \subset \widehat{L}_{i}^{u}$ at each point $x \in \widehat{l_{i}^{s}} \cap \widehat{l}_{i}^{u}$ we define $\vec{v}_{x}^{s}\left(\vec{v}_{x}^{u}\right)$ as a tangent vector to $\widehat{l}_{i}^{s}\left(\widehat{l}_{i}^{u}\right)$ at the point $x$, directed according to the orientation $\widehat{l}_{i}^{s}\left(\widehat{l}_{i}^{u}\right)$. Then we define an index $\xi_{x}$ of the point $x$ equal to $1(-1)$, if the orientation that an ordered pair of vectors $\left(\vec{v}_{x}^{u}, \vec{v}_{x}^{s}\right)$ defines on the manifold $\widehat{V}_{i}$, coincides (does not coincide) with the orientation of the manifold $\widehat{V}_{i}$. Then we will call the number

$$
\xi_{i}=\sum_{x \in \hat{l}_{i}^{s} \cap \widehat{l}_{i}^{u}} \xi_{x}
$$

by the index of heteroclinic intersection of curves $\widehat{l}_{i}^{s}$ and $\widehat{l}_{i}^{u}$. The index of the heteroclinic intersection of curves coincides for any two curves $\widehat{l}_{i}^{s}$ and $\widehat{l}_{i}^{u}$ from the sets $\widehat{L}_{i}^{s}$ and $\widehat{L}_{i}^{u}$ respectively. We state

$$
\xi_{F}=\sum_{i=1}^{m_{0}} \xi_{i}
$$

and will call the number $\xi_{F}$ by the heteroclinic intersection index of the diffeomorphism $F \in$ $\in M S_{1}\left(\mathbb{T}^{2}\right)$. The following result shows that the heteroclinic intersection index uniquely determines the type of the homotopy class $[f]$ of the diffeomorphism $f \in M S_{1}\left(\mathbb{T}^{2}\right)$.

Theorem 1. Let $f \in M S_{1}\left(T^{2}\right)$ and $F=f^{d}$. The homotopy class $[f]$ is of type $T_{1}$, if and only if $\xi_{F}=0$. The homotopy class $[f]$ is of type $T_{2}$, if and only if $\xi_{F} \neq 0$. 


\section{Proof of the Lemma 1}

Let $f \in M S_{1}\left(\mathbb{T}^{2}\right), F=f^{d}: \mathbb{T}^{2} \rightarrow \mathbb{T}^{2}$ and all heteroclinic domains $V_{i}, i \in\left\{1, \ldots, m^{\prime}\right\}$ of the diffeomorphism $F$ are trivial. Then the curves $b_{i}, i \in\left\{1, \ldots, m^{\prime}\right\}$ bound the disks $D_{i} \subset \mathbb{T}^{2}$ such that $F\left(D_{i}\right) \subset \operatorname{int}\left(D_{i}\right)$ or $F^{-1}\left(D_{i}\right) \subset \operatorname{int}\left(D_{i}\right)$. Since the domain $V_{i}$ contains heteroclinic points, then $D_{i}$ contains at least one saddle point. By virtue of $[9 \text {, Lemma } 6.3, \text { p. 1115 }]^{1}$ there is an isotopy $H_{t}: \mathbb{T}^{2} \rightarrow \mathbb{T}^{2}, t \in[0,1]$, such that $H_{0}=F, H_{1}=F_{1}$, where $F_{1}: \mathbb{T}^{2} \rightarrow \mathbb{T}^{2}$ Morse-Smale diffeomorphism coinciding with $F$ on the set $\mathbb{T}^{2} \backslash\left(D_{1} \cup \ldots \cup D_{m^{\prime}}\right)$ and such that each disk $D_{i}$, $i \in\left\{1, \ldots, m^{\prime}\right\}$ contains a single fixed sink or source of the diffeomorphism $F_{1}$ (see Fig. 2). By construction, the diffeomorphism $F_{1}$ is a gradient-like diffeomorphism, all non-wandering points of which are fixed and have a positive orientation type. According to [10, Theorem 4.2], the diffeomorphism $F$ is isotopic to the identity map. Since $F=f^{d}$, then $f_{*}^{d}=i d$. Thus, the matrix $f_{*}$ is periodic and, therefore, the diffeomorphism $[f]$ has the homotopy type $T_{1}$.

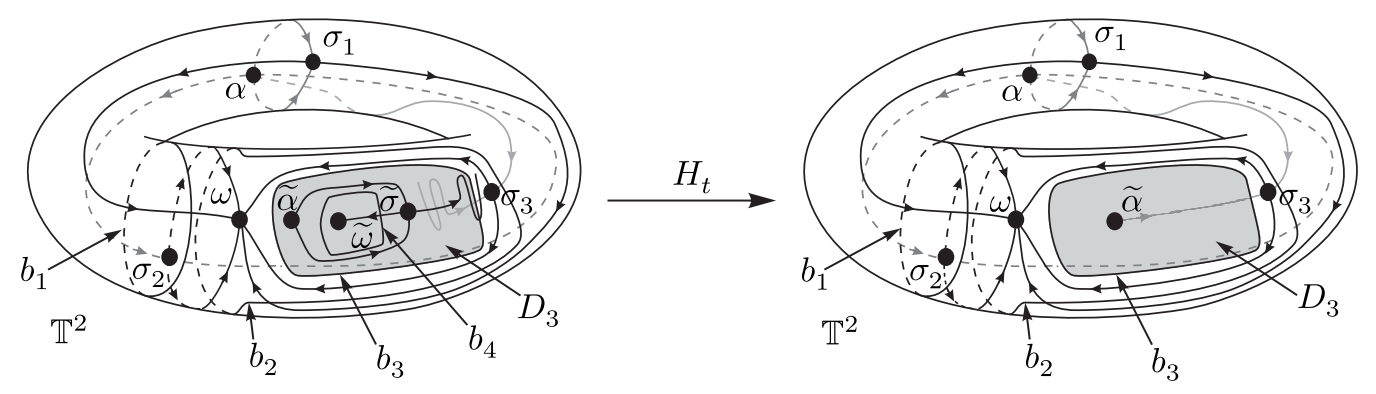

Fig. 2. An example of the application of the lemma 1. On the left is the phase portrait of the diffeomorphism $F$ on the torus $\mathbb{T}^{2}$ with marked curves $b_{i}, i \in\{1, \ldots, 4\}$ and the disk $D_{3}$ containing the repeller connected component $\mathcal{R}_{f}$. On the right is a diffeomorphism $F_{1}$ on the torus $\mathbb{T}^{2}$, which coincides with the diffeomorphism $F$ everywhere except for the disk $D_{3}$, on which, using the isotopy, we trivialize the connected component of the repeller to the only sink $\widetilde{\alpha}$

\section{Proof of the Theorem 1}

Let $f \in M S_{1}\left(\mathbb{T}^{2}\right)$ and $F=f^{d}$. Without loss of generality, we will assume that $V_{i}=S^{1} \times \mathbb{R}$, $\left.F\right|_{V_{i}}(s, r)=(s, r+1)$ and $K_{i}=S^{1} \times[0,1]$. We state $U_{i}^{+}=S^{1} \times[0,+\infty)$.

Consider the set $E_{F}$ of such diffeomorphisms $G: \mathbb{T}^{2} \rightarrow \mathbb{T}^{2}$ such that $G$ coincides with $F$ in some neighborhood $U_{G}^{+}$of the attractor $\mathcal{A}_{F}$, containing $\bigcup_{i=1}^{m_{0}} K_{i}$, in some neighborhood $U_{G}^{-}$of the repeller $\mathcal{R}_{F}$ and $\Omega_{F}=\Omega_{G}$. For any diffeomorphism $G \in E_{F}$ we denote by $L_{G, i}^{s}$ and $L_{G, i}^{u}$ the set of stable and unstable saddle separatrices of the diffeomorphism $G$, that belong to the set $V_{i}$. Then each connected component of the orbit spaces $L_{G, i}^{s} / G$ and $L_{G, i}^{u} / G$ is homeomorphic to a circle. Since $K_{i}$ is a common fundamental domain of diffeomorphisms $\left.G\right|_{V_{i}}$ and $\left.F\right|_{V_{i}}$, then $L_{G, i}^{s} / G$ is

\footnotetext{
${ }^{1}$ Lemma 6.3 from [9] asserts that any gradient-like diffeomorphism $f$ on the surface $M^{2}$, with an attractor $A$ belonging to the disk $D$ and such that $f(D) \subset D, A=\bigcap_{n \in \mathbb{N}} f^{n}(D)$, is connected by a stable arc (diffeotopy admitting only flip and saddle knot bifurcations) with some gradient-like diffeomorphism $g$, coinciding with $f$ on $M^{2} \backslash D$ and having a single non-wandering point in the disk $D$, which is a fixed sink.
} 
homeomorphic to $p_{i}\left(L_{G, i}^{s} \cap K_{i}\right)$ and $L_{G, i}^{u} / G$ is homeomorphic to $p_{i}\left(L_{G, i}^{u} \cap K_{i}\right)$. Since $\left.G\right|_{U_{i}^{+}}=$ $=\left.F\right|_{U_{i}^{+}}$, then $L_{G, i}^{s} \cap U_{i}^{+}=\bigcup_{n \geqslant 0} F^{n}\left(L_{G, i}^{s} \cap K_{i}\right), L_{G, i}^{u} \cap U_{i}^{+}=\bigcup_{n \geqslant 0} F^{n}\left(L_{G, i}^{u} \cap K_{i}\right)$ and, therefore, $p_{i}\left(L_{G, i}^{s} \cap K_{i}\right)=p_{i}\left(L_{G, i}^{s} \cap U_{i}^{+}\right), p_{i}\left(L_{G, i}^{u} \cap K_{i}\right)=p_{i}\left(L_{G, i}^{u} \cap U_{i}^{+}\right)$. Let

$$
\widehat{L}_{G, i}^{s}=p_{i}\left(L_{G, i}^{s} \cap U_{i}^{+}\right), \quad \widehat{L}_{G, i}^{u}=p_{i}\left(L_{G, i}^{u} \cap U_{i}^{+}\right) .
$$

Let us prove an auxiliary lemma.

Lemma 2. Let $\widehat{h}: \widehat{V}_{i} \rightarrow \widehat{V}_{i}$ is isotopic to the identity diffeomorphism. Then there exists a diffeotopy $\zeta_{t} \subset E_{F}$ such that $\zeta_{0}=F, \zeta_{1}=F_{1}$ and $\widehat{L}_{F_{1}, i}^{u}=\widehat{h}\left(\widehat{L}_{F, i}^{u}\right), \widehat{L}_{F_{1}, i}^{s}=\widehat{L}_{F, i}^{s}$.

Proof. Take an open cover $D=\left\{\widehat{D}_{1}, \ldots, \widehat{D}_{q}\right\}$ of the manifold $\widehat{V}_{i}$ by two-dimensional disks so that $p_{i}^{-1}\left(\widehat{D}_{j}\right) \cap S^{1} \times\left\{r_{j}, r_{j}+1\right\}=\varnothing$ for some $r_{j} \in \mathbb{R}$. According to [11, Fragmentation lemma] there exist diffeomorphisms $\widehat{w}_{1}, \ldots, \widehat{w}_{q}: \widehat{V}_{i} \rightarrow \widehat{V}_{i}$ smoothly isotopic to the identity and with the following properties:

i) for each $j \in\{1, \ldots, q\}$ there is a smooth isotopy $\left\{\widehat{w}_{j, t}\right\}$, identical outside $\widehat{D}_{j}$ and joining the identity map and $\widehat{w}_{j}$;

ii) $\widehat{h}=\widehat{w}_{1} \ldots \widehat{w}_{q}$.

Let $U_{G, i}^{+}=V_{i} \cap U_{G}^{+}, U_{G, i}^{-}=V_{i} \cap U_{G}^{-}$and denote by $r_{G}^{-}, r_{G}^{+}$real positive numbers such that $S^{1} \times\left(-\infty,-r_{G}^{-}\right) \subset U_{G, i}^{-}$and $S^{1} \times\left(-r_{G}^{+},+\infty\right) \subset U_{G, i}^{+}$.

Take $q$ disks $D_{1}, \ldots, D_{q}$ one in each of the sets $p_{i}^{-1}\left(\widehat{D}_{1}\right), \ldots, p_{i}^{-1}\left(\widehat{D}_{q}\right)$ so that $D_{j} \subset \widetilde{K}_{j}$, with $\widetilde{K}_{j}=S^{1} \times\left(-r_{j}-1,-r_{j}\right), r_{G}^{-}<r_{1}$ and $r_{j}+1<r_{j+1}$ for $j \in\{1, \ldots, q-1\}$. Let $w_{j, t}: V \rightarrow V$ be a diffeomorphism that coincides with $\left(\left.p_{i}\right|_{\widetilde{K}_{j}}\right)^{-1} \widehat{w}_{j, t} p_{i}$ on the set $\widetilde{K}_{j}$ and coincides with the identity map outside the set $\widetilde{K}_{j}$. Let

$$
\zeta_{t}=w_{1, t} \ldots w_{q, t} F .
$$

By the construction $\zeta_{t} \subset E_{f}$ and $\zeta_{0}=F$. Let $F_{1}=\zeta_{1}$. Let us show that $\widehat{L}_{F_{1}, i}^{u}=\widehat{h}\left(\widehat{L}_{F, i}^{u}\right)$, $\widehat{L}_{F_{1}, i}^{s}=\widehat{L}_{F, i}^{s}$.

Since the manifolds $L_{F_{1}, i}^{s}$ are obtained from the local manifolds $L_{F_{1}, i}^{s} \cap U_{F_{1}, i}^{+}$by iteration due to the map $F_{1}^{-1}$ and $L_{F_{1}, i}^{s} \cap U_{F_{1}, i}^{+}=L_{F, i}^{s} \cap U_{F_{1}, i}^{+}$, then $L_{F_{1}, i}^{s} \cap U_{i}^{+}=L_{F, i}^{s} \cap U_{i}^{+}$and therefore $\widehat{L}_{F_{1}, i}^{s}=$ $=\widehat{L}_{F, i}^{s}$.

We state $\widetilde{U}_{i}^{-}=S^{1} \times\left(-\infty,-r_{q}-1\right)$ and $F_{j}=w_{j} \ldots w_{q} F, j \in\{1, \ldots, q\}$. By the construction $F_{j} \in E_{F}$. Then, to prove the lemma, it suffices to show that $\widehat{L}_{F_{j}, i}^{u}=\widehat{w}_{j}\left(\widehat{L}_{F_{j+1}, i}^{u}\right)$, where $F_{q+1}=F$.

Take natural numbers $k_{j}^{-}, k_{j}^{+}$such that $\widetilde{K}_{j}^{-}=F_{j}^{-k_{j}^{-}}\left(\widetilde{K}_{j}\right) \subset \widetilde{U}_{i}^{-}, \widetilde{K}_{j}^{+}=F_{j}^{k_{j}^{+}}\left(\widetilde{K}_{j}\right) \subset U_{i}^{+}$. We state $k_{j}=k_{j}^{-}+k_{j}^{+}$. Since the manifolds $L_{F_{j}, i}^{u}$ are obtained from the local manifolds $L_{F_{j+1}, i}^{u} \cap U_{F_{j}}^{-}$ by iteration due to the map $F_{j}$ and $L_{F_{j}, i}^{u} \cap \widetilde{U}_{i}^{-}=L_{F_{j+1}, i}^{u} \cap \widetilde{U}_{i}^{-}$, then (see Fig. 3)

$$
\begin{aligned}
\widehat{L}_{F_{j+1}, i}^{u} & =p_{i} F_{j+1}^{k_{j}}\left(L_{F_{j+1}, i}^{u} \cap \widetilde{K}_{j}^{-}\right), \\
\widehat{L}_{F_{j}, i}^{u} & =p_{i} F_{j}^{k_{j}}\left(L_{F_{j+1}, i}^{u} \cap \widetilde{K}_{j}^{-}\right) .
\end{aligned}
$$



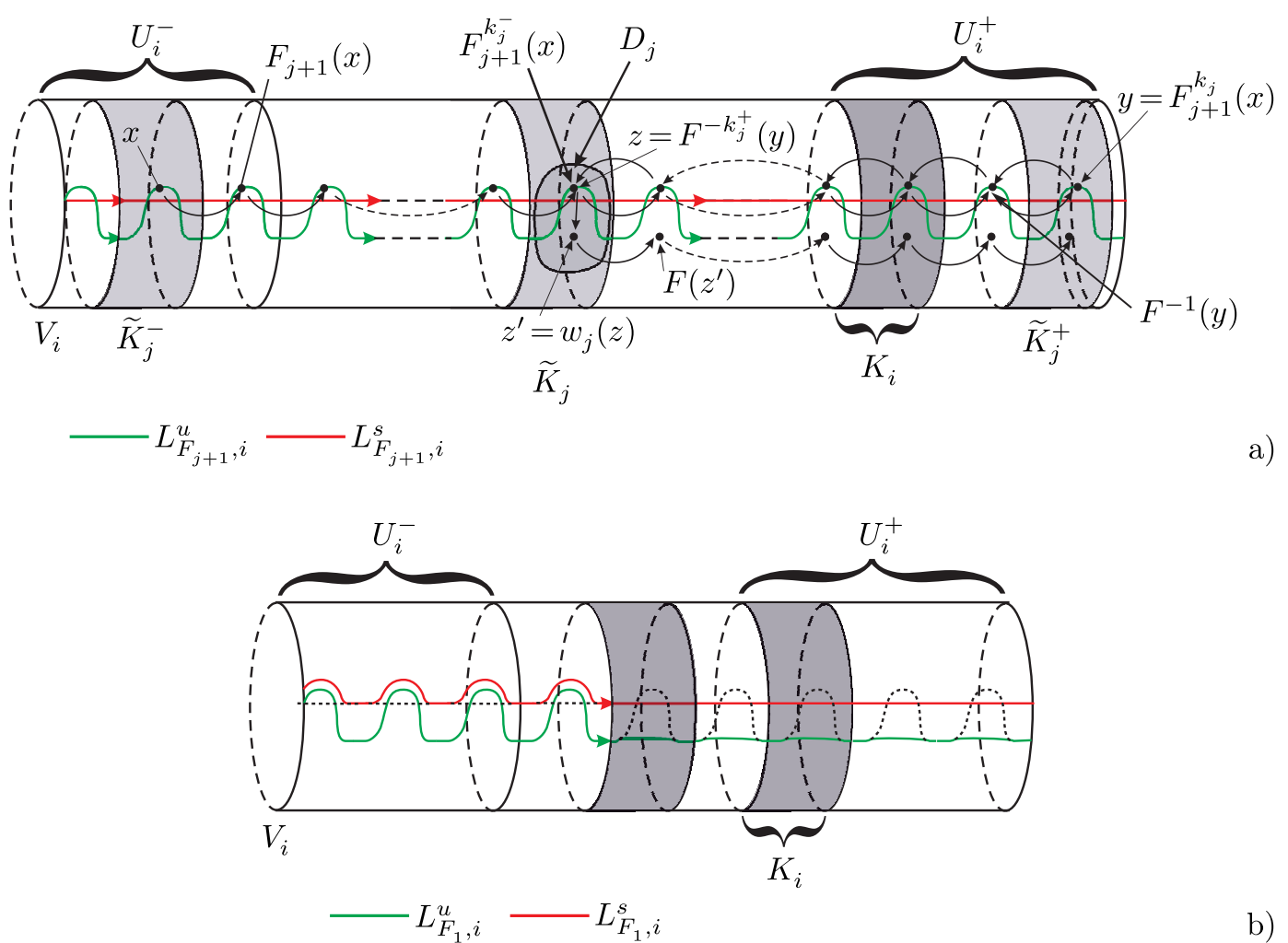

b)

Fig. 3. The figure (a) shows an illustration of the action of the formula step by step. $x \underset{F_{j+1}^{k_{j}}}{\longrightarrow} y \underset{F^{-k_{j}^{+}}}{\longrightarrow}$ $\underset{F^{-k_{j}^{+}}}{\longrightarrow} z \underset{w_{j}}{\longrightarrow} z^{\prime} \underset{F^{-k_{j}^{+}}}{\longrightarrow} F^{k_{j}^{+}}\left(z^{\prime}\right)$. The figure (b) shows the form of separates $L_{F_{1}, i}^{s}, L_{F_{1}, i}^{u}$ for the diffeomorphism $F_{1}$

Then

$$
\begin{aligned}
& \widehat{L}_{F_{j}, i}^{u}=p_{i} F_{j+1}^{k_{j}^{+}} w_{j} F_{j+1}^{k_{j}^{-}}\left(L_{F_{j+1}, i}^{u} \cap \widetilde{K}_{j}^{-}\right)= \\
&=p_{i} F^{k_{j}^{+}} w_{j} F^{-k_{j}^{+}} F_{j+1}^{k_{j}}\left(L_{F_{j+1}, i}^{u} \cap \widetilde{K}_{j}^{-}\right)=p_{i} F^{k_{j}^{+}} w_{j} F^{-k_{j}^{+}}\left(\left.p_{i}\right|_{\widetilde{K}_{j}^{+}}\right)^{-1} p_{i} F_{j+1}^{k_{j}}\left(L_{F_{j+1}, i}^{u} \cap \widetilde{K}_{j}^{-}\right)= \\
& \quad=p_{i} w_{j}\left(\left.p_{i}\right|_{\widetilde{K}_{j}}\right)^{-1}\left(\widehat{L}_{F_{j+1}, i}^{u}\right)=\widehat{w}_{j}\left(\widehat{L}_{F_{j+1}, i}^{u}\right) .
\end{aligned}
$$

Let's return to the proof of the theorem. By assumption, the diffeomorphism $F$ has nontrivial heteroclinic domains $V_{i}, i \in\left\{1, \ldots, m_{0}\right\}$ and curves from the sets $\widehat{L}_{F, i}^{s}$ and $\widehat{L}_{F, i}^{u}$ have homotopy types $\left\langle\delta_{i}, d_{i}^{s}\right\rangle$ and $\left\langle\delta_{i}, d_{i}^{u}\right\rangle$ respectively on the torus $\widehat{V}_{i}$. Then, by virtue of [8, Chapter D, Exercise 7] the equality $\xi_{i}=\delta_{i}\left(d_{i}^{u}-d_{i}^{s}\right)$ is holds.

We define the function $\nu:[0,1] \rightarrow[0,1]$ by the formula

$$
\nu(t)= \begin{cases}0, & t=0, \\ \frac{1}{1+\exp \left(\frac{\frac{1}{2}-t}{t^{2}(t-1)^{2}}\right)}, & 0<t<1, \\ 1, & t=1 .\end{cases}
$$


Define on the annuli $K_{i}$ the map $R_{i}: K_{i} \rightarrow K_{i}$, by the formula

$$
R_{i}\left(e^{i \phi}, r\right)=\left(e^{i\left(\phi+2 \pi \xi_{i} \nu(r)\right)}, r\right)
$$

The projection $\widehat{R}_{i}=p_{i} R_{i} p_{i}^{-1}$ of the map $R_{i}$ acts on the fundamental group of the torus $\widehat{V}_{i}$ by the matrix $\widehat{R}_{i *}$. Due to the consistency of the bases $a, b$ and $\widehat{a}_{i}, \widehat{b}_{i}$, the equality $\widehat{R}_{i *}=R_{i *}$ holds. Then, the diffeomorphism $R_{i}$ acts on the fundamental group of the torus $\mathbb{T}^{2}$ by the matrix $R_{i *}=$ $=\left(\begin{array}{cc}1 & \xi_{i} \\ 0 & 1\end{array}\right)$. Let $\widetilde{F}=R_{m_{0}}^{-1} \circ \ldots \circ R_{1}^{-1} \circ F$. Then $\widetilde{F} \in E_{F}$ and for any $i \in\left\{1, \ldots, m_{0}\right\}$ the equality holds

$$
\left(\begin{array}{ll}
\delta_{i} & d_{i}^{u}
\end{array}\right)\left(\begin{array}{cc}
1 & -\xi_{i} \\
0 & 1
\end{array}\right)=\left(\begin{array}{ll}
\delta_{i} & d_{i}^{s}
\end{array}\right) .
$$

Since the global stable manifold of a saddle point is obtained from a local one by iteration due to the inverse map and in a neighborhood of the attractor $\mathcal{A}_{F}$ the manifolds $L_{i}^{s}$ and $L_{\widetilde{F}, i}^{s}$ are coincide, then $L_{\widetilde{F}, i}^{s} \cap K_{i}=L_{i}^{s} \cap K_{i}$. Since the global unstable manifold of a saddle point is obtained from the local by iterating by the direct map and in the neighborhood of the repeller $\mathcal{R}_{F}$ the manifolds $L_{i}^{u}$ and $L_{\widetilde{F}, i}^{u}$ are coincide, then $L_{\widetilde{F}, i}^{u} \cap K_{i}=R_{i}\left(L_{i}^{u} \cap K_{i}\right)$.

Thus, the knots $\widehat{L}_{\widetilde{F}, i}^{s}, \widehat{L}_{\widetilde{F}}^{u}, i$ have the same homotopy type on the torus $\widehat{V}_{i}$. By virtue of [8], there exists a diffeomorphism $\widehat{h}: \widehat{V}_{i} \rightarrow \widehat{V}_{i}$ isotopic to the identity such that $\widehat{h}\left(\widehat{L}_{\widetilde{F}, i}^{u}\right) \cap \widehat{L}_{\widetilde{F}, i}^{s}=\varnothing$. According to the lemma 2, the diffeomorphism $\widetilde{F}$ is diffeotopic to some diffeomorphism $\widetilde{F}_{1} \in E_{F}$ such that $\widehat{L}_{\widetilde{F}_{1}, i}^{u} \cap \widehat{L}_{\widetilde{F}_{1}, i}^{s}=\varnothing$. By construction, the diffeomorphism $\widetilde{F}_{1}$ belongs to the class $M S\left(\mathbb{T}^{2}\right)$ and has only trivial heteroclinic domains (see Fig. 4).
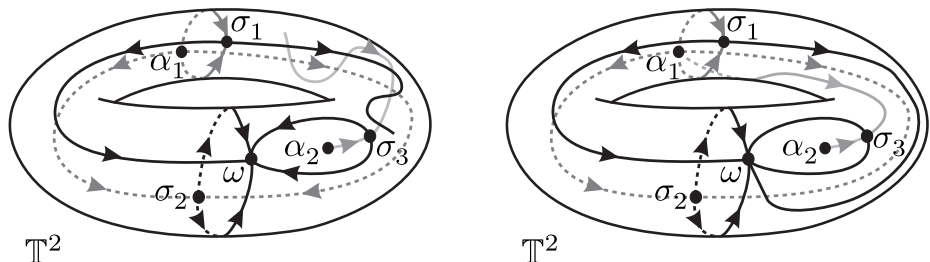

Fig. 4. On the left is the phase portrait of the diffeomorphism $\widetilde{F}$ on the torus $\mathbb{T}^{2}$ with a non-trivial heteroclinic domain, $\widetilde{F}$ is reduced by some isotopy to the diffeomorphism $\widetilde{F}_{1}$, the phase portrait of which is shown on the right

By virtue of the lemma 1 , the diffeomorphism $\widetilde{F}_{1}$ is isotopic to the identity diffeomorphism. Then the diffeomorphism $F$ is isotopic to the diffeomorphism $R_{1} \circ \ldots \circ R_{m_{0}}$. Because

$$
R_{i *}=\left(\begin{array}{cc}
1 & \xi_{i} \\
0 & 1
\end{array}\right),
$$

then

$$
F_{*}=\left(\begin{array}{cc}
1 & \xi_{F} \\
0 & 1
\end{array}\right)
$$

Then $F$ belongs to the homotopy type $T_{1}$ if and only if $\xi_{F}=0$. Since $F=f^{d}$, then the matrix $f_{*}$ is periodic if and only if $\xi_{F}=0$. Thus, the diffeomorphism $[f]$ has homotopy type $T_{1}$ if and only if $\xi_{F}=0$. 
Since the Morse - Smale diffeomorphisms are representatives of only homotopy classes from $T_{1}$ and $T_{2}$, then $[f] \in T_{2}$ if and only if $\xi_{F} \neq 0$.

\section{Acknowledgments}

The author thanks V.Z. Grines and O. V. Pochinka for formulation of the problem and fruitful discussions.

\section{References}

[1] Thurston, W. P., On the Geometry and Dynamics of Diffeomorphisms of Surfaces, Bull. Amer. Math. Soc. (N.S.), 1988, vol. 19, no. 2, pp. 417-431.

[2] Farb, B. and Margalit, D., A Primer on Mapping Class Groups, Princeton Math. Ser., vol. 49, Princeton, N.J.: Princeton Univ. Press, 2012.

[3] Smale, S., Differentiable Dynamical Systems, Bull. Amer. Math. Soc., 1967, vol. 73, no. 6, pp. 747817.

[4] Bezdenezhnykh, A.N. and Grines, V.Z., Dynamical Properties and Topological Classification of Gradient-Like Diffeomorphisms on Two-Dimensional Manifolds: 1, Selecta Math. Soviet., 1992, vol. 11, no. 1, pp. 1-11.

[5] Grines, V.Z., Morozov, A.I., and Pochinka, O.V., Realization of Homeomorphisms of Surfaces of Algebraically Finite Order by Morse - Smale Diffeomorphisms with Orientable Heteroclinic Intersection, Tr. Mat. Inst. Steklova, 2021, vol. 315, pp. 95-107 (Russian).

[6] Grines, V.Z., Zhuzhoma, E. V., Medvedev, V.S., and Pochinka, O. V., Global Attractor and Repeller of Morse-Smale Diffeomorphisms, Proc. Steklov Inst. Math., 2010, vol. 271, no. 1, pp. 103-124; see also: Tr. Mat. Inst. Steklova, 2010, vol. 271, pp. 111-133.

[7] Grines, V.Z., Pochinka, O.V., and Van Strien, S., On 2-Diffeomorphisms with One-Dimensional Basic Sets and a Finite Number of Moduli, Mosc. Math. J., 2016, vol. 16, no. 4, pp. 727-749.

[8] Rolfsen, D., Knots and Links, Math. Lecture Ser., vol. 7, Houston: Publish or Perish, Inc., 1990.

[9] Nozdrinova, E. and Pochinka, O., Solution of the 33rd Palis-Pugh Problem for Gradient-Like Diffeomorphisms of a Two-Dimensional Sphere, Discrete Contin. Dyn. Syst., 2021, vol. 41, no. 3, pp. 1101-1131.

[10] Palis, J., On Morse-Smale Dynamical Systems, Topology, 1968, vol. 8, no. 4, pp. 385-404.

[11] Banyaga, A., On the Structure of the Group of Equivariant Diffeomorphisms, Topology, 1977, vol. 16, no. 3, pp. 279-283. 